\title{
Galectin-3: A Heart Failure Biomarker as Sign of Active Coronary Heart Disease
}

\author{
Ivica Bošnjak ${ }^{*}$, Dražen Bedeković², Kristina Selthofer-Relatić1,3, Ines Bilić-Ćurčić1,4 \\ ${ }^{1}$ Clinic of Internal Medicine, Department of Cardiovascular Disease and Intensive Care, University Hospital Centre Osijek, \\ Osijek, Croatia \\ ${ }^{2}$ Department of Internal Medicine, General Hospital Našice, Našice, Croatia \\ ${ }^{3}$ Department of Internal Medicine and Medical Ethics, School of Medicine, University Josip Juraj Strossmayer, Osijek, Croatia \\ ${ }^{4}$ Department of Pathophysiology, School of Medicine, University Josip Juraj Strossmayer, Osijek, Croatia \\ Email: *ivica_bosnjak@net.hr
}

How to cite this paper: Bošnjak, I., Bedeković, D., Selthofer-Relatić, K. and Bilić-Ćurčić, I. (2017) Galectin-3: A Heart Failure Biomarker as Sign of Active Coronary Heart Disease. World Journal of Cardiovascular Diseases, 7, 373-379.

https://doi.org/10.4236/wjcd.2017.711035

Received: September 14, 2017

Accepted: November 5, 2017

Published: November 8, 2017

Copyright $\odot 2017$ by authors and Scientific Research Publishing Inc. This work is licensed under the Creative Commons Attribution-NonCommercial International License (CC BY-NC 4.0). http://creativecommons.org/licenses/by-nc/4.0/

\begin{abstract}
Atherosclerosis is characterized by the accumulation of cholesterol esters, macrophages and fibrous elements on the inner artery wall. This process begins with accumulation of plasma lipoproteins on the inner wall of the artery, which leads to changes in the passage and elasticity of the blood vessels. Monocytes penetrating the arterial wall transforms into macrophages which digest cholesterol and form foam cells which is one of the first steps in atherosclerotic process. Activation of macrophages is affected by galectin-3, a $\beta$-galactoside-binding lectin which is also involved in cardiac remodeling. Cardiac matrix remodeling is the ultimate result of macrophages proliferation and chemotaxis, neutrophil extravasation, oxidative stress, apoptosis, angiogenesis, fibroblast proliferation and deposition of collagen. Studies show that elevated levels of galectin 3 within atherosclerotic lesions in humans are closely related to the development of a disease itself. With this review, we want to demonstrate the correlation between galectin- 3 which is precipitated in atherosclerotic plaque and has an influence on the development of cardiovascular diseases and its role in the prognosis of recovery in cardiac patients.
\end{abstract}

\section{Keywords}

Galectin-3, Atherosclerosis, Coronary Artery Disease,

Coronary Heart Disease, Macrophages, Heart Failure

\section{Introduction}

Atherosclerosis is systematic inflammatory disease which affects major arteries including coronary arteries. The essence of pathophysiological process lies in 
forming atherosclerotic lesions, which includes: accumulation of cholesterol esters, monocyte-macrophage migration and accumulation of fibrous elements in vessel intimal layer. Rupture of such lesion results in thrombus formation and vessel lumen obstruction causing myocardial infarction, cerebral insult or peripheral artery disease. Macrophages have an important role in this process. Accumulation of macrophage foam cells in atherosclerotic plaques results in predominantly from macrophage proliferation which migrates in affected area [1].

Galectin-3 (Gal-3) is a member of a galectin family involved in numerous physiological and pathological processes such as inflammation and formation of fibrous tissue [2]. It is found in a wide range of tissues and is essential for normal macrophage functioning [3] [4]. In animal model, adding a granulocyte-macrophage colony stimulating factor resulted in 6-fold higher expression of Gal-3 following macrophages activation and it can be concluded that Gal-3 has dependent stimulatory effect on macrophage [2] [5]. Several authors have proved an association between Gal-3 level, cardiac fibrosis and remodeling. Galectin-3 is also a marker of heart failure, regardless of left ventricle ejection fraction. It was next logical step to investigate association between Gal-3 level and atherosclerosis. Nachtigal reported an increased level of Gal-3 in atherosclerotic plaques [6]. Gal-3 level is much higher in patients with unstable coronary disease, which leads to conclusion that Gal-3 can be involved in atherosclerotic plaque destabilization [7].

\section{Submolecular Moments of Galectin-3 Pathophysiology in Atherosclerosis}

Atherosclerosis, as a systemic disease, can't be observed only trough classic risk factors such as smoking, hypertension, diabetes mellitus or dyslipidemia. This is complicated process where monocyte-macrophages and inflammatory cytokines play an important role.

Despite efforts to keeping risk factors under control, many patients have recurrent vascular events as a result of ongoing atherosclerotic process, increased activity of monocyte-macrophage system and increased level of galectin-3. Inflammation and oxidative stress are underlying mechanism of Gal-3 involvement in atherosclerotic process. Galectin-3 expression is enhanced in macrophages and vessels smooth muscle cells to mediate foam cells development [8]. Figure 1 represents potential mechanism of galectin-3 role in atherosclerotic process.

Madrigal-Matute in his study showed that phorbol myristate acetate (PMA) induce Gal-3 expression and mediate in model of THP-1 in macrophage differentiation. On the other hand, apocynin reverse this effect indicating that Gal-3 induction by PMA depends on NADPH/ROS system [9]. Gal-3 also participates in the production of superoxide in monocytes implying that Gal-3 is part of closed circuit between oxidative stress and inflammation process [10]. There is observed correlation between Gal-3 level and NADPH oxidase-dependent superoxide production in asymptomatic patients diagnosed with atherothrombosis. 


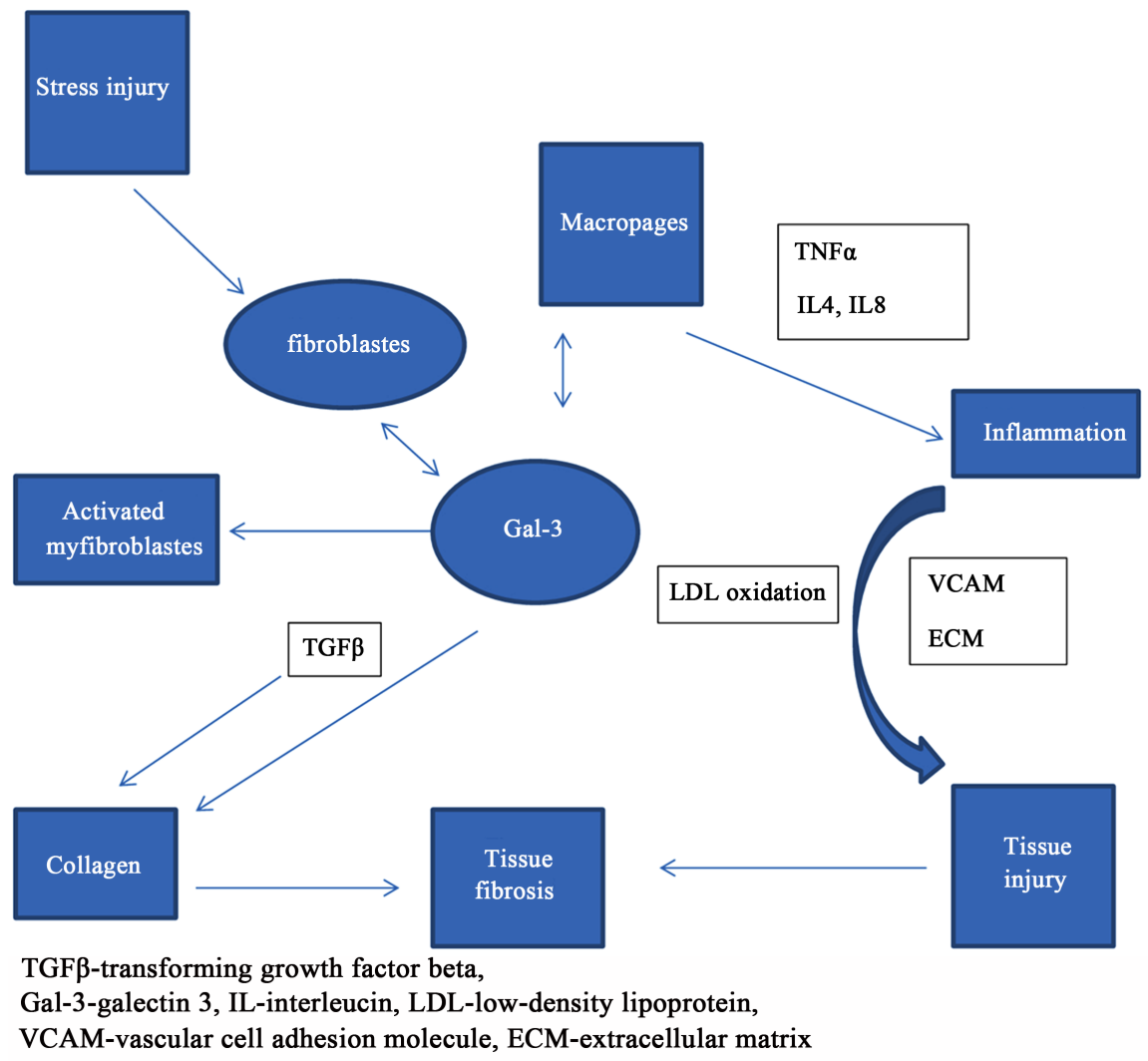

Figure 1. Galectin-3 role in atherosclerotic process.

Besides NADPH, other enzymes also plays an important role in atherosclerotic processes (lipoxygenase, xanthine oxidase, NO synthase) [11].

Galectin-3 can be intracellular or extracellular. Intracellular Gal-3 participates in cells signalization and migration, as well as in apoptosis. Extracellular Gal-3 intermediates adhesion, cytokine production, chemo adhesion and receptor function. Monocytes relies Gal-3 which increase exosomes expression mediated by ROS. IncreasedGal-3 plasma levels are founded in patients exosomes suffering from atherosclerosis, even asymptomatic [9].

As already mentioned before, Gal-3 is chemo attractant for monocyte-macrophages cells, therefore it's possible that Gal-3 participate in atherosclerotic plaque progression. Gal-3 stimulate, in vitro and in vivo, monocyte/macrophage chemotaxis [12].

MacKinnon et al. in their study reported reduced plaque formation when Gal-3 is deleted from the outset, despite high cholesterol intake and high serum cholesterol level [12]. Authors propose strategy of blocking a galectin-3, resulting of plaque formation inhibition without affecting plaque stability [12]. Authors used a modified citrus pectin (MCP), a natural pectin found in the citrus fruit peel and pulp. MCP inhibits Gal-3 function in vitro and in vivo [13] [14] [15]. In animal model of $\mathrm{ApoE}^{-/-}$mice with high cholesterol intake administration of $1 \%$ MCP reduced plaque progression compared with placebo. Mice deficient in Gal-3 had a reversal effect on iNOS and arginase activity, reduced ma- 
crophages M2 activity and reduced atherosclerotic plaque progression [12].

\section{Clinical Aspects of Galectin-3 in Coronary Artery Disease}

Galectin-3 is well investigated in heart failure (HF) pathophysiology, and has diagnostic and prognostic value. In contrast to other HF markers, such as NTproBNP or troponin, galectin-3 doesn't show a fluctuation in serum level, once elevated remains increased in majority of cases and isn't affected by standard HF medical treatment [16].

Clinical Gal-3 significance inhuman atherosclerotic process is less known. Despite this, there are articles reporting importance and association between galectin-3 and atherosclerosis in humans.

According to available studies data, Gal-3 level correlate with sex, body mass index, diuretic therapy usage, triglycerides serum level, homocystein plasma level and is inversely proportional to glomerular filtration rate [17]. Homocystein stimulates activity of nuclear factor $\mathrm{kB}$ - an inducer of Gal-3, therefore homocystein lowering agents can contribute to lowering Gal-3 level [18] [19].

One of the indirect evidence of galectin-3 pivotal role in the inflammation and atherosclerosis process is presented by Tsail et al. study. Compared to healthy patients control group, patients diagnosed with ST segment elevation myocardial infarction (STEMI) had much higher Gal-3 level, and higher WBC count-as index of inflammation [20]. Also, multivessel coronary patients had higher level of Gal-3 than control group, and multi vessel disease correlates with higher Gal-3 level and WBC count [20]. Considering the fact those were STEMI patients, many with multi vessel disease, we can conclude Gal-3 plays an important role in inflammation process and atherosclerotic plaque progression, but it predict also instability and possible plaque rupture [20]. Same study has confirmed that patients with Gal-3 higher level had worse clinical presentation, higher Killip and CADILLAC score and higher indication for IABP usage Gal-3. Elevated Gal-3 can be an indicator for heart failure development rafter AMI and is strong predictor of 30-day major adverse events for patients with STEMI undergoing primary percutaneous coronary intervention [20].

Higher serum level of Gal-3 is observed in patients diagnosed with unstable coronary heart disease (CHD). There is a significant association between Gal-3 serum level and number of affected coronary blood vessels [7]. Long term follow up has shown Gal-3 as a cardiovascular events and mortality predictor for patients diagnosed with CHD [17] [21]. High Gal-3 level is present in patients with carotid atherosclerotic disease and strongly correlates with intima media thickness ratio [9]. In patients with type 2 diabetes mellitus (DMT2), the degree of CHD was evaluated with CT angiography is compared to Gal-3 serum level. It is confirmed by this trial that higher level of Gal-3 strongly correlate with CHD, total number of affected arteries, number of plaques and calcified plaque types [22]. Kusaka had similar result for patients with CHD without DMT2 [23]. In the same article, authors have found high Gal-3 serum level association with a Gensini score, which implies a strong relationship between Gal-3 and severity of 
CHD [23]. Observed significant association between serum Gal-3 level and hs-CRP, as marker of inflammatory atherosclerotic process [24], number of affected coronary arteries, Gensini score, WBC could be a marker of severity of CHD, supporting of Gal-3 major role in atherosclerotic process [25].

\section{Conclusion}

It is only a matter of time before galectin-3 becomes a reality in everyday clinical practice. As one of the new markers for heart failure, galectin- 3 is mentioned in the new ESC guidelines for the heart failure treatment. Galectin-3 measurement may be a significant factor in making a treatment decisions in coronary heart disease and heart failure, but also in heart failure caused by coronary heart disease. Gal-3 levels are directly associated with severity of CHD, as well as with remodeling and fibrotic process in the myocardium, which implicate an importance of galectin-3 in both pathophysiological processes. Future investigations are needed to assess utility of galectin-3 for prediction of atherosclerotic disease as well as heart failure development. Gal-3 can be used as a target molecule for development of disease modifying agent which could have a direct impact on the pathological processes of $\mathrm{CHD}$ and $\mathrm{HF}$, affecting the stability of the disease, quality of life, need for hospitalization and revascularization in those patients.

\section{References}

[1] Robbins, C.S., Hilgendorf, I., Weber, G.F., Theurl, I., Iwamoto, Y., Figueiredo, J.L., Gorbatov, R., Sukhova, G.K., Gerhardt, L.M., Smyth, D., Zavitz, C.C., Shikatani, E.A., Parsons, M., van Rooijen, N., Lin, H.Y., Husain, M., Libby, P., Nahrendorf, M., Weissleder, R. and Swirski, F.K. (2013) Local Proliferation Dominates Lesional Macrophage Accumulation in Atherosclerosis. Nature Medicine, 19, 1166-1172. https://doi.org/10.1038/nm.3258

[2] Bošnjak, I., Selthofer-Relatić, K. and Včev, A. (2015) Prognostic Value of Galectin-3 in Patients with Heart Failure. Disease Markers, 2015, Article ID: 690205. https://doi.org/10.1155/2015/690205

[3] Dumic, J., Dabelic, S. and Flögel, M. (2006) Galectin-3: An Open Ended Story. Biochimica et Biophysica Acta, 1760, 616-635.

[4] Sano, H., Hsu, D.K., Apgar, J.R., Yu, L., Sharma, B.B., Kuwabara, I., Izui, S. and Liu, F.T. (2003) Critical Role of Galectin-3 in Phagocytosis by Macrophages. Journal of Clinical Investigation, 112, 389-397. https://doi.org/10.1172/JCI200317592

[5] Elliott, M.J., Strasser, A. and Metcalf, D. (1991) Selective Up-Regulation of Macrophage Function in Granulocyte-Macrophage Colony-Stimulating Factor Transgenic Mice. Journal of Immunology, 147, 2957-2963.

[6] Nachtigal, M., Al-Assaad, Z., Mayer, E.P., Kim, K. and Monsigny, M. (1998) Galectin-3 Expression in Human Atherosclerotic Lesions. American Journal of Pathology, 152, 1199-1208.

[7] Falcone, C., Lucibello, S., Mazzucchelli, I., Bozzini, S., D’Angelo, A., Schirinzi, S., Totaro, R., Falcone, R., Bondesan, M. and Pelissero, G. (2011) Galectin-3 Plasma Level and Coronary Artery Disease: A New Possible Biomarjer of Acute Coronary Syndrome. International Journal of Immunopathology and Pharmacology, 24, 905-913. https://doi.org/10.1177/039463201102400409 
[8] Rong, J.X., Shapiro, M., Trogan, E. and Fisher, E.A. (2003) Transdifferentiation of Mouse Aortic Smooth Muscle Cells to Macrophage-Like State after Cholesterol Loading. Proceedings of the National Academy of Sciences of the United States of America, 100, 13531-13536. https://doi.org/10.1073/pnas.1735526100

[9] Madrigal-Matute, J., Lindholt, J.S., Fernandez-Garcia, C.E., Benito-Martin, A., Burillo, E., Zalba, G., Beloqui, O., Llamas-Granda, P., Ortiz, A., Egido, J., Blanco-Colio, L.M. and Martin-Ventura, J.L. (2014) Galectin-3, Biomarker Linking Oxidative Stress and Inflammation with the Clinical Outcomes of Patients with Atherothrombosis. Journal of the American Heart Association, 3, pii: e000785.

https://doi.org/10.1161/JAHA.114.000785

[10] Suzuki, Y., Inoue, T., Yoshimaru, T. and Ra, C. (2008) Galectin-3 But No Galectin-1 Induces Mast Celldeath by Oxidative Stress and Mitochondrial Permeability Transition. Biochimica et Biophysica Acta, 1783, 924-934.

[11] Madamanchi, N.R., Vendrov, A. and Runge, M.S. (2005) Oxidative Stress and Vascular Disease. Arteriosclerosis, Thrombosis, and Vascular Biology, 25, 29-38.

[12] MacKinnon, A.C., Liu, X., Hadoke, P.W.F., Miller, M.R., Newby, D. and Sethi, T. (2013) Inhibition of Galectin. 3 Reduced Atherosclerosis in Apolipoprotein E-Deficient Mice. Glycibiology, 23, 654-663. https://doi.org/10.1093/glycob/cwt006

[13] Pienta, K.J., Naik, H., Akhtar, A., Yamazaki, K., Replogle, T.S., Lehr, J., Donat, T.L., Tait, L., Hogan, V. and Raz, A. (1995) Inhibition of Spontaneus Metastasis in a Rat Prostae Cancer Model by Oral Administartion of Modified Citrus Pectin. Journal of the National Cancer Institute, 87, 348-353. https://doi.org/10.1093/jnci/87.5.348

[14] Nangia-Makker, P., Hogan, V., Honjo, Y., Baccarini, S., Tait, L., Bresalier, R. and Raz, A. (2002) Inhibition of Human Cancer Cell Growth and Metastasis in Nude Mice by Oral Intake of Modified Citrus Pectin. Journal of the National Cancer Institute, 94, 1854-1862. https://doi.org/10.1093/jnci/94.24.1854

[15] Liu, H.Y., Huang, Z.L., Yang, G.H., Lu, W.Q. and Yu, N.R. (2008) Inhibitory Effect of Modified Citrus Pectin on Liver Metastasis in a Mouse Colon Cancer Model. World Journal of Gastroenterology, 14, 7386-7391. https://doi.org/10.3748/wjg.14.7386

[16] Lok, D.J., Van der Meer, P., de la Porte, P.W., Lipsic, E., Van Wijngaarden, J., Hillege, H.L. and van Veldhuisen, D.J. (2010) Prognostic Value of Galectin-3, a Novel Marker of Fibrosis, in Patients with Chronic Heart Failure: Data from the DEAL-HF Study. Clinical Research in Cardiology, 99, 323-328. https://doi.org/10.1007/s00392-010-0125-y

[17] Maiolino, G., Rossito, G., Pedon, L., Cesari, M., Frigo, A.C., Azzolini, M., Plebani, M. and Rossi, G.P. (2015) Galectin-3 Predicts Long-Term Cardiovascular Death in High-Risk Patient with Coronary Artery Disease. Arteriosclerosis, Thrombosis, and Vascular Biology, 35, 725-732. https://doi.org/10.1161/ATVBAHA.114.304964

[18] Wang, G., Siow, Y.L. and O, K. (2000) Homocysteine Stimulate Nucler Factor kappaB Activity and Monocyte Chemoattractant Protein-1 Expression in Vascular Smooth and Monocyte Cells: Possible Role of Protein Kinase C. Biochemical Journal, 352, 817-826. https://doi.org/10.1042/bj3520817

[19] Dumic, J., Lauc, G. and Flögel, M. (2000) Expression of Galectin-3 in Cells Exposed to Stress-Roles of Jun and NF-kappaB. Cellular Physiology and Biochemistry, 10, 149-158. https://doi.org/10.1159/000016345

[20] Tsai, T.H., Sung, P.H., Chang, L.T., Sun, C.K., Yeh, K.H., Chung, S.Y., Chua, S., Chen, Y.L., Wu, C.J., Chang, H.W., Ko, S.F. and Yip, H.K. (2012) Value and Level of Galectin-3 in Acute Myocardial Infarction Patients Undergoing Primary 
Percutaneous Coronary Intervention. Journal of Atherosclerosis and Thrombosis, 19, 1073-1082. https://doi.org/10.5551/jat.12856

[21] Tuñón, J., Blanco-Colio, L., Cristóbal, C., Tarín, N., Higueras, J., Huelmos, A., Alonso, J., Egido, J., Asensio, D., Lorenzo, Ó., Mahíllo-Fernández, I., Rodríguez-Artalejo, F., Farré, J., Martín-Ventura, J.L. and López-Bescós, L. (2014) Usefullness of a Combination of Monocyte Chemoattractant Protein-1, Galectin-3 and N-Terminal Probrain Natriuretic Peptide to Predict Cardiovascular Events in Patients with Coroanry Artery Disease. American Journal of Cardiology, 113, 434-440.

[22] Ozturk, D., Celik, O., Satilmis, S., Aslan, S., Erturk, M., Cakmak, H.A., Kalkan, A.K., Ozyilmaz, S., Diker, V. and Gul, M. (2015) Association between Serum Galectin-3 Levels and Coronary Atherosclerosis and Plaque Burden/Structure in Patients with Type 2 Diabetes Mellitus. Coronary Artery Disease, 26, 396-401. https://doi.org/10.1097/MCA.0000000000000252

[23] Kusaka, H., Yamamoto, E., Hirata, Y., Fujisue, K., Tokitsu, T., Sugamura, K., Sakamoto, K., Tsujita, K., Kaikita, K., Hokimoto, S., Sugiyama, S. and Ogawa, H. (2015) Clinical Significance of Plasma Galectin-3 in Patinets with Coronary Artery Disease. International Journal of Cardiology, 201, 532-534.

[24] De Boer, R.A., van Veldhuisen, D.J., Gansevoort, R.T., Muller Kobold, A.C., van Gilst, W.H., Hillege, H.L., Bakker, S.J. and van der Harst, P. (2012) The Fibrosis Marker Galectin-3 and Outcome in the General Popilation. Journal of Internal Medicine, 272, 55-64. https://doi.org/10.1111/j.1365-2796.2011.02476.x

[25] Aksan, G., Gedikli, O., Keskin, K., Nar, G., Inci, S., Yildiz, S.S., Kaplan, Ö., Soylu, K., Kiliçkesmez, K.O. and Șahin, M. (2016) Is Galectin-3 a Biomarker, a Player or Both in the Precence of Coronary Atherisclerosis? Journal of Investigative Medicine, 64, 764-770. 